\title{
Prevalence and Antimicrobial Susceptibility Pattern of Mono therapy and combination therapy of Cefepime in Pseudomonas aeruginosa isolates of patients from a tertiary care hospital in Karachi, Pakistan
}

\author{
Kanaan Mansoor ${ }^{1}$, Syed Bilal Tanvir' ${ }^{1}$, Ali Shariq, Saba Shahnawaz ${ }^{2}$, Sumera Ahmed ${ }^{1}$
}

\section{Kanaan Mansoor ${ }^{1}$, Syed Bilal Tanvir ${ }^{1}$, Ali Shariq, Saba Shahnawaz', Sumera Ahmed $^{1}$}

${ }^{1}$ Dr. Ziauddin University Hospital, Karachi, PAKISTAN.

${ }^{2}$ Medical Officer, The Kidney Centre, Karachi, PAKISTAN.

\section{Correspondence}

Kanaan Mansoor, Department, Dr. Ziauddin University Hospital Karachi, Pakistan.

Phone numbers +923002334895

Email: kanaanm@gmail.com

\section{History}

- Submission Date: 03-09-15;

- Review completed: 18-06-16;

- Accepted Date: 22-06-16.

DOI : 10.5530/ijmedph.2016.3.4

Article Available online

http://www.ijmedph.org/v6/i3

\section{Copyright}

(C) 2016 Phcog.Net. This is an open-access article distributed under the terms of the Creative Commons Attribution 4.0 International license.

\begin{abstract}
Introduction: Pseudomonas aeruginosa is a bothersome pathogen on the rise and prone to developing resistance during treatment. Hospitalized patients are especially prone to its detrimental effects.

Aims: To determine the prevalence and antimicrobial susceptibility pattern of Mono therapy and combination therapy of Cefepime in Pseudomonas aeruginosa isolates obtained from patients at a tertiary care hospital in Karachi, Pakistan.

Methods: This study was conducted at a university-affiliated, urban teaching hospital. During a 2-year period (January 2013 to December 2015), all hospitalized patients with a positive blood culture for $P$. aeruginosa were eligible for this investigation, amounting to a sample size of 634, and a cross sectional study was performed. Standard microbiological methods were used to identify the clinical isolates. The isolates were cultured on chocolate and MacConkey agar.

Results: Throughout the duration of this study, 634 isolates of $P$. aeruginosa were cultured. Positive cultures were then tested against the following drugs: Cefepime, Meropenem, Amikacin and Ciprofloxacin. Cefepime was $76.2 \%$ (483) sensitive for isolates while the age and sex relationship analysis showed that isolates gathered from 0-18 year old females were $94.6 \%$ sensitive to Cefepime. Around $63.25 \%$ isolates were sensitive to the combination of Cefepime and Amikacin while the combination offering the least resistance was that of Cefepime and Ciprofloxacin (7.1\%).

Conclusions: $P$. aeruginosa isolates show a progressive trend of resistance to Cefepime. Cefepime when used in combination with Ciprofloxacin, potentially will be more effective than monotherapy with Cefepime.

Key words: P. aeruginosa, Cefepime, B-lactam, Susceptibility pattern, Antimicrobials, Karachi.
\end{abstract}

\section{INTRODUCTION}

Pseudomonas aeruginosa has emerged as quite a challenging pathogen for clinicians. Resistant strains of $P$. aeruginosa were first detected in Western Europe in the 1980s, and then in 1991 they were reported in Japan, leading this to become a growing concern worldwide. ${ }^{1}$ Pseudomonas aeruginosa bacteremia occurs most frequently in critically ill patients, particularly those who are immunocompromised such as cystic fibrosis patients, burn victims and ICU patients. ${ }^{2,3}$ It stands to reason then that there is an increased predisposition amongst hospitalized HIV positive patients for contracting this pathogen as demonstrated by Tacconelli E et al. ${ }^{5}$ Chronic $P$. aeruginosa infections lead to lung tissue destruction which ultimately results in an untimely death for the patient ${ }^{4}$ P. aeruginosa demonstrates staggering statistics when it comes to nosocomial infections; being the $2^{\text {nd }}$ most common pathogen to cause pneumonia, $8^{\text {th }}$ most common for bacteremia, and $3^{\text {rd }}$ most common for urinary tract infections as demonstrated by the Center of Disease Control and Prevention (CDC), Nosocomial Infection Surveillance System in the United States of America. ${ }^{6}$ With this pathogen contributing $10-20 \%$ to nosocomial infections, it is regarded as the bane of a hospitalized patient.

The Carbapenems, namely Imipenem and Meropenem were known for their effectuality against this dreaded pathogen, but recent studies have shown that the efficacy of these drugs has decreased due to a growing resistance to these compounds, which is a cause of apprehension for clinicians. ${ }^{7}$ Since $P$. aeruginosa develops resistance to antibiotics during the course of treatment ${ }^{8}$ and is also intrinsically resistant to a myriad of drugs owing to the 
broadly specific drug efflux pump and its synergy with the low degree of outer membrane permeability, ${ }^{10,11}$ it is quite a vexing pathogen.

Cefepime is a $4^{\text {th }}$ gen cephalosporin that has a broad spectrum antibacterial activity. Its coverage is better than $3^{\text {rd }}$ gen cephalosporins ${ }^{10}$ which is why Cefepime was recommended as a treatment of pneumonia and cystic fibrosis. ${ }^{12,13}$ The objective of the current study was to determine the anti microbial susceptibility patterns of Mono therapy and combination therapy using Cefepime against Pseudomonas aeruginosa isolates obtained from patients at a tertiary care hospital in Karachi, Pakistan.

\section{MATERIAL AND METHODS}

This cross sectional study was conducted at the Microbiology Department of Ziauddin Medical University Hospital Karachi, Pakistan from January 2013 to January 2015, over a period of two years. Patient records from the microbiology department were used to gather the data and a total of 634 patients from all age groups had a positive culture for $P$. aeruginosa. These cultures were obtained from samples of tracheal aspirate, blood, pus, body fluids, urine and broncoalveolar lavage.

The samples were cultured on Chocolate and MacConkey agar and incubated at $37^{\circ} \mathrm{C}$ for a duration of 18 hours. The isolates were identified according to standard microbiological methods. ${ }^{14}$ P. aeruginosa by its colony morphology, on gram staining shows up as a pleomorphic gram negative rod. On MacConkey agar it shows up as a non-lactose fermenter which produces a pigment that has a characteristic grape like odor. $P$. aeruginosa is oxidase positive and a non-motile bacterium which has the ability to reduce nitrate to nitrite. Api $20 \mathrm{NE}$ was used for the confirmation of isolates.

The antibiotic sensitivity patterns of these isolates were studied by using the Kirby Bauer Disc Diffusion method on Mueller-Hinton agar, in accordance with CLSI 2014 Guidelines, ${ }^{15}$ and using Hi-media antibiotic discs. The antibiotics which were tested included, Amikacin $(30 \mathrm{mcg})$, Cefepime $(30 \mathrm{mcg})$, Ciprofloxacin $(5 \mathrm{mcg})$ and Meropenem $(10 \mathrm{mcg})$. Strains which had similar resistance patterns (antibiotype) were considered to be from the same clone. The Pseudomonas aeruginosa ATCC 27853 strain was used for quality control during this study.

Statistical analysis was performed using SPSS version 20 and the prevalence and susceptibility pattern of Cefepime for P. aeruginosa was calculated.

\section{RESULTS}

From January 2013 till January 2015, a total of 634 patients from all age groups were included in this study since they had a positive culture for $P$. aeruginosa. They were further divided into 3 categories of ages 0-18 years, 19-60 years and 60 years and above. Table 1 shows the distribution of samples between different age groups and sex.

We found Cefepime to be sensitive in $76.2 \%$ (483) of cases and $23.8 \%$ resistant. Cefepime was found to be the most sensitive drug amongst female patients aged 0-18 years, being effective for $94.6 \%$ of the cases, while it was least sensitive in males over the age of 60 , around $73.3 \%$. Thus the pattern that emerges, indicates that increasing age shows an increased resistance to this drug. Table 2. Shows the susceptibility pattern according to sex amongst different age groups, indicating a progressive decrease in sensitivity with age.

Tables 3 show the susceptibility pattern of $P$. aeruginosa when treated with combinations of Cefepime with Amikacin, Meropenem and Ciprofloxacin respectively.

Table 3 shows that Cefepime, a $4^{\text {th }}$ gen cephalosporin has a different sensitivity pattern against $P$. aeruginos $a$ when used in combination with other antibiotics. The most sensitive combination was found to be that of Cefepime and Amikacin, being 63.25\% sensitive, followed by Cefepime and Meropenem at a sensitivity of 59.78\%, and Cefepime and Cipro- floxacin being $49.2 \%$ sensitive. The least resistive pattern found was that of the combination of Cefepime and Ciprofloxacin (7.1\%), followed by Cefepime and Amikacin (16.5\%) and the most resistance was noted for the combination of Cefepime and Meropenem (17.5\%). Tables 4-6 show different combination therapies with their sensitivity and resistance patterns according to age and sex. Our data strongly supports the use of Cefepime and Ciprofloxacin as combination therapy on account of its low resistance to the isolates.

\section{DISCUSSION}

Pseudomonas aeruginosa is a non-fermenting, gram negative, pleomorphic bacillus which is freely found in nature and the environment (ubiquitous). P. aeruginosa gives rise to a multitude of nosocomial infections ranging from UTI, to pneumonia and septicemia.

In our study we found Cefepime to be sensitive in $76.2 \%$ (483) which was slightly higher than the one reported in Pakistan by Ahmad Ullah H. et al in 2013. Their study showed this drug to be resistant in $15 \%$ of the isolates. ${ }^{19}$ Our figures were also in stark contrast to a study done in Urmia, Iran, where Cefepime was found to be $75.4 \%$ resistant, with $22.4 \%$ of the isolates found to be of intermediate resistance and $2.1 \%$ isolates showing sensitivity to Cefepime. ${ }^{16}$ From India, Patel et al reported Cefepime to be $15.63 \%$ resistant in isolates of $P$. aeruginos $a^{17}$ where as Endimiani et al reported that $10-35 \%$ of the isolates of the clinical population in North America are resistant to Cefepime. ${ }^{18}$

It has been strongly recommended that when Pseudomonas aeruginosa is the insinuated culprit, combination therapy trumps mono therapy due to the various resistance mechanisms in action, namely porin channel mutations, bacterial efflux pumps, alteration in the target site of the antibiotic, loss of membrane proteins, IMP-type metalloenzymes or carbapenemases etc. ${ }^{20,21}$ Assorted variations of combination therapies are standard for different sites of infection. Patients suffering from VAP and neutropenia are treated rigorously with anti Pseudomonal drugs in order to treat or further prevent Pseudomonal infections. In such patients, Piperacillin/Tazobactum in combination with Amikacin was proposed for the treatment while in another study, Levofloxacin (Fluoroquinolone) in combination with Cefepime was recommended, ${ }^{22,23}$ this is in concordance with our study which showed that the most effective treatment that can be proposed would be Ciprofloxacin (Fluoroquinolone) and Cefepime because of its low resistance of 7.1\% . Drago L et al demonstrated that the synergistic effect of combination therapy against $P$. aeruginosa showed there was enhanced activity when Fluoroquinolones were used in conjunction with B-Lactams and Amikacin. ${ }^{24}$

Various combination of antibiotics have been used for the treatment of P. aeruginosa such as B-lactams and fluoroquinolones or B-lactams and aminoglycosides ${ }^{25}$ and the appropriate concoction of drugs should be administered to the patient as soon as possible since a delay in combination therapy has been known to cause an increase in mortality. ${ }^{26}$ A meta-analysis reported that in conditions where there is a blood stream infection of $P$. aeruginosa, only combination therapy can yield favorable results. ${ }^{27}$ B-lactams and fluoroquinolones are known for their effect on $P$. aeruginosa because of their excellent penetration in different sites of the body as well as being less nephrotoxic, but with the use of fluoroquinolones there is a concurrent rise in C. difficile infections. ${ }^{28}$

There is an increasing trend of increased Cefepime resistance in Pakistan as shown by our study when compared with a study done previously. Awareness should be spread about $P$. aeruginosa being a diverse organism that is able to gain resistance while being treated, so that physicians make it a point to use combination therapy as opposed to mono therapy, when they suspect $P$. aeruginosa infections in patients. 
Table 1:'Age and Sex distribution of sample'

\begin{tabular}{ccc}
\hline Age Group & No. of Male Patients $(\mathrm{N}=368)$ & No. of Female Patients $(\mathrm{N}=266)$ \\
\hline $0-18$ years $(\mathrm{N}=90)$ & $53(8.36 \%)$ & $37(5.8 \%)$ \\
$19-60$ years $(\mathrm{N}=257)$ & $139(21.92 \%)$ & $118(18.61 \%)$ \\
60 years and above $(\mathrm{N}=287)$ & $176(27.7 \%)$ & $111(17.51 \%)$ \\
\hline
\end{tabular}

Table 2: 'Susceptibility pattern of Cefepime'

\begin{tabular}{ccccccc}
\hline \multicolumn{3}{c}{ MALE } & \multicolumn{3}{c}{ FEMALE } \\
\hline & $0-18$ & $19-60$ & 60 above & $0-18$ & $19-60$ & 60 above \\
\hline Sensitive & $40(75.5 \%))$ & $103(74.1 \%)$ & $129(73.3 \%)$ & $35(94.6 \%)$ & $92(77.9 \%)$ & $84(75.7 \%)$ \\
Resistance & $13(24.5 \%)$ & $36(25.9 \%)$ & $47(26.7 \%)$ & $2(5.41 \%)$ & $26(22.0 \%)$ & $27(24.3 \%)$ \\
\hline
\end{tabular}

Table 3: 'Cefepime combined with Amikacin, Meropenem and Ciprofloxacin' combination

\begin{tabular}{cccc}
\hline & \multicolumn{3}{c}{ CEFEPIME } \\
\hline \multirow{3}{*}{ Amikacin } & & Sensitive & Resistant \\
& Sensitive & $401(63.25 \%)$ & $46(7.25 \%)$ \\
& Resistant & $82(12.93 \%)$ & $105(16.56 \%)$ \\
Meropenem & & Sensitive & Resistant \\
& Sensitive & $379(59.8 \%)$ & $40(6.31 \%)$ \\
Ciprofloxacin & Resistant & $104(16.4 \%)$ & $111(17.5 \%)$ \\
& & Sensitive & Resistant \\
& Sensitive & $312(49.2 \%)$ & $106(16.7 \%)$ \\
& Resistant & $171(27 \%)$ & $45(7.1 \%)$ \\
\hline
\end{tabular}

Table 4: 'Sensitivity and Resistance Pattern of Cefepime with Amikacin against $P$. aeruginosa'

\begin{tabular}{ccccc}
\hline Age & Sex & Sensitivity & Resistance & Intermediate Resistance \\
\hline \multirow{3}{*}{$0-18$} & Male $(\mathrm{N}=53)$ & $62.3 \%(\mathrm{n}=33)$ & $16.9 \%(\mathrm{n}=9)$ & $20.7 \%(\mathrm{n}=11)$ \\
& Female $(\mathrm{N}=37)$ & $62.2 \%(\mathrm{n}=23)$ & $2.7 \%(\mathrm{n}=1)$ & $35.1 \%(\mathrm{n}=13)$ \\
\multirow{2}{*}{$19-59$} & Male $(\mathrm{N}=139)$ & $64.02 \%(\mathrm{n}=89)$ & $15.1 \%(\mathrm{n}=21)$ & $20.8 \%(\mathrm{n}=29)$ \\
& Female $(\mathrm{N}=118)$ & $61.01 \%(\mathrm{n}=72)$ & $12.7 \%(\mathrm{n}=15)$ & $26.2 \%(\mathrm{n}=31)$ \\
\multirow{3}{*}{60 and above } & Male $(\mathrm{N}=176)$ & $64.8 \%(\mathrm{n}=114)$ & $21.5 \%(\mathrm{n}=38)$ & $13.6 \%(\mathrm{n}=24)$ \\
& Female $(\mathrm{N}=111)$ & $63.1 \%(\mathrm{n}=70)$ & $18.9 \%(\mathrm{n}=21)$ & $18.01 \%(\mathrm{n}=20)$ \\
\hline
\end{tabular}

Table 5: 'Sensitivity and Resistance Pattern of Cefepime with Ciprofloxacin against $P$. aeruginosa'

\begin{tabular}{ccccc}
\hline Age & Sex & Sensitivity & Resistance & Intermediate Resistance \\
\hline \multirow{2}{*}{$0-18$} & Male $(\mathrm{N}=53)$ & $47.25 \%(\mathrm{n}=25)$ & $5.6 \%(\mathrm{n}=3)$ & $47.25 \%(\mathrm{n}=25)$ \\
& Female $(\mathrm{N}=37)$ & $67.5 \%(\mathrm{n}=25)$ & $0 \%(\mathrm{n}=0)$ & $32.4 \%(\mathrm{n}=12)$ \\
\multirow{2}{*}{$19-59$} & Male $(\mathrm{N}=139)$ & $47.5 \%(\mathrm{n}=66)$ & $7.9 \%(\mathrm{n}=11)$ & $44.6 \%(\mathrm{n}=62)$ \\
& Female $(\mathrm{N}=118)$ & $56.8 \%(\mathrm{n}=67)$ & $5.9 \%(\mathrm{n}=7)$ & $37.29 \%(\mathrm{n}=44)$ \\
\multirow{2}{*}{60 and above } & Male $(\mathrm{N}=176)$ & $43.2 \%(\mathrm{n}=76)$ & $10.2 \%(\mathrm{n}=18)$ & $46.5 \%(\mathrm{n}=82)$ \\
& Female $(\mathrm{N}=111)$ & $47.7(\mathrm{n}=53)$ & $5.4 \%(\mathrm{n}=6)$ & $46.8 \%(\mathrm{n}=52)$ \\
\hline
\end{tabular}

Table 6: 'Sensitivity and Resistance Pattern of Cefepime with Meropenem against P. aeruginosa'

\begin{tabular}{ccccc}
\hline Age & Sex & Sensitivity & Resistance & Intermediate Resistance \\
\hline \multirow{2}{*}{$0-18$} & Male $(\mathrm{N}=53)$ & $58.5 \%(\mathrm{n}=31)$ & $18.9 \%(\mathrm{n}=10)$ & $22.6 \%(\mathrm{n}=12)$ \\
& Female $(\mathrm{N}=37)$ & $62.2 \%(\mathrm{n}=23)$ & $2.7 \%(\mathrm{n}=1)$ & $35.1 \%(\mathrm{n}=13)$ \\
\multirow{2}{*}{$19-59$} & Male $(\mathrm{N}=139)$ & $59.7 \%(\mathrm{n}=83)$ & $18.7 \%(\mathrm{n}=26)$ & $21.6 \%(\mathrm{n}=30)$ \\
& Female $(\mathrm{N}=118)$ & $60.1 \%(\mathrm{n}=71)$ & $11.0 \%(\mathrm{n}=13)$ & $28.8 \%(\mathrm{n}=34)$ \\
\multirow{2}{*}{60 and above } & Male $(\mathrm{N}=176)$ & $58.5 \%(\mathrm{n}=103)$ & $22.2 \%(\mathrm{n}=39)$ & $19.3 \%(\mathrm{n}=34)$ \\
\hline
\end{tabular}




\section{CONCLUSION}

Cefepime for many years has been considered to be one of the most reliable drugs that can safely be used for patients with $P$. aeruginosa infections. There is a progressive trend that shows that $P$. aeruginosa is found be resistant to this antibiotic now. Thus this awareness should be promptly spread so that physicians administer prompt combination therapy. We suggest that a combination of Cefepime and Ciprofloxacin is the most effective therapy to battle this pathogen. More research is warranted so that new drugs are discovered to deal with $P$. aeruginosa infections effectively.

\section{ACKNOWLEDGEMENT}

We offer our sincerest gratitude to Dr. Sumera Sheikh, Dr Arif Hussain, Dr. Badar Jamal and Dr.Adnan Zubairi for their continuous support and mentorship throughout the conception and execution of this project.

\section{CONFLICT OF INTEREST}

The author declare no conflict of interest.

\section{REFERENCES}

1. Sacife W, Youngs HR, Paton Rh and Amyes Sg. Transferable imipenemreistance in acinetobacter species from a clinical source. J Antimicrob Chemother 1995:36(3):585-6.

2. Carmeli YN, Troillet G, Eliopoulos GM and Samore MH. Emergence of antibiotic resistance Pseudomonas aeruginosa: Comparison of risk associated with different antipseudomonal agents. Antimicrob Agents Chemother. 1999;43(6):1379-82

3. Gilligan PH. Microbiology of airway disease in patients with cystic fibrosis. Clin Microbiol Rev. 1991;4(1):35-51.

4. Webb AK. (1995). The treatment of pulmonary infection cystic fibrosis. Scand J Infect Dis. 1995;96(Supp I):24-7.

5. Tacconelli E, Tumbarello M, Bertagnolio S, Citton R, Spanu T, Fadda G, et al. Multidrug-Resistant Pseudomonas aeruginosa Bloodstream Infections: Analysis of Trends in Prevalence and Epidemiology. Emerg Infect Dis. 2002;8(2):220-1.

6. Elizabeth $\mathrm{BH}$, Vincent HT. Impact of multi-drug resistant Pseudomonas aeruginosa infection on patient outcomes. Expert Rev Pharmacoecon Outcomes Res. 2010;10(4):441451.

7. Liver more, DM. of Pseudomonas, porins, pumps and carbapenems.J Antimicrob Chemother. 2001;47:247-50.

8. Breidenstein EB, de la Fuente-Nunez C, Hancock RE: Pseudomonas aeruginosa: all roads lead to resistance. Trends Microbiol. 2011;19(8):419-26.

9. Pai H, Kim JW, Kim J, Lee JH, Choe KW, Gotoh N. Carbepenem resistance mechanism in Pseudomonas aeruginosa clinical isolates. Antimicrob Agents Chemother. 2001;45(2):480-4.

10. Gad GF, El- Domany RA, Zaki S, Ashour HM.2007 characterization of Pseudomonas aeruginosa isolated from clinical and environmental samples in minia Egypt: Prevalance, Antibiogram and Resistance Mechanism. J Antimicrob Che- mother 2007:60:1010-7.

11. Barradell LB Bryson HM. Cefepime. A review of its antibacterial activity, pharmacokinetic properties and therapeutic use. Drugs. 1994;47:471-505.

12. Chapman TM, Perry CM. Cefepime: a review of its use in the management of hospitalized patients with pneumonia. Am J Respir Med. 2003;2:75-107.

13. Bosso JA, Saxon BA, and Matsen JM. Comparative activity of cefepime, alone and in combination, against clinical isolates of Pseudomonas aeruginosa and Pseudomonas cepacia from cystic fibrosis patients. Antimicrob. Agents Chemother. 1991;35:783-4.

14. Koneman E, Winn WJ, Allen S, Janda W, Procop G, Woods G, et al. Koneman's color atlas and textbook of diagnostic microbiology. Sixth edition, Chapter Lippicott Williams \& Wilkins, London. 2006;6:264-9

15. Clinical and Laboratory Standards Institute (CLSI).2011 Performance standards for antimicrobials susceptibility testing: Twenty First informational supplement. M100-S21. Wayne, PA:CLSI:2011.

16. 1 Jazani $\mathrm{NH}$, Babazadeh $\mathrm{H}$, Sabahi $\mathrm{Z}$ and Zartoshti $\mathrm{M}$. The evaluation of antibiotic resistance to cefepime in hospital isolates of Pseudomonas Aeruginosa. J Med Biomed Sci. 2010;9:17-21.

17. Patel $\mathrm{MH}$, Trivedi GR, Patel SM and Vegad MM. Antibiotic susceptibility pattern in urinary isolates of gram negative bacilli with special reference to AmpC $\beta$-lactamase in a tertiary care hospital. Urol Ann. 2010;2(1):7-11.

18. Endimiani, F. Perez F. and R.A. Bonomo. Cefepime: a reappraisal in an era of increasing antimicrobial resistance. Expert Rev Anti Infect Ther. 2008;6(6):805-24

19. Ahmad Ullah $\mathrm{H}$, Khan Khalid, Jamil Sahrish, Taj Ayaz, AnwerAtif. In vitro compar ative studies on susceptibility patterns of Pseudomonas aeruginosa and Staphylococcus aureus to Cefepime and Cefipirome. Int Res J Pharm. 2013,4(5): $137-40$

20. Nordmann P, Poirel L. Emerging carbapenemases in gram-negative aerobes Clin Microbiol Infect. 2002;8:321-31.

21. P A Lambert Mechanisms of antibiotic resistance in Pseudomonas aeruginosa. J R Soc Med. 2002;95(Suppl 41):22-6.

22. Fowler RA, Flavin KE, Barr J, Weinacker AB, Parsonnet J, Gould MK. Variability in antibiotic prescribing patterns and outcomes in patients with clinically suspected ventilator-associated pneumonia. Chest. 2003;123(3):835-44

23. Ahmed SM, Choudhary J, Ahmed M, Arora V, Parul, Ali S. Treatment of ventilator-associated pneumonia with piperacillin-tazobactum and amikacin vs cefepime and levofloxacin: A randomized prospective study. Indian J Crit Care Med. 2007;11:117-21.

24. Drago L, De Vecchi E, Nicola L, Colombo A, Guerra A, Gismondo M.R. Activity of Levofloxacin and Ciprofloxacin in Combination with Cefepime, Ceftazidime Imipenem, Piperacillin-Tazobactam and Amikacin against Different Pseudomonas aeruginosa Phenotypes and spp. Chemotherapy. 2004;50(4):202-10.

25. Bradley JS, Jackson MA. The use of systemic and topical fluoroquinolones.Pediatrics. 2011.128:e1034-45

26. Ibrahim EH, Sherman G, Ward S, Fraser VJ, Kollef MH. 2000. The influence of inadequate antimicrobial treatment of bloodstream infections on patient outcomes in the ICU setting.Chest. 2000;118(1):146-55.

27. Safdar N, Handelsman J, Maki DG. 2004. Does combination antimicrobial therapy reduce mortality in Gram-negative bacteraemia? A metaanalysis. Lancet Infect Dis. 2004;4(8):519-27.

28. Pepin J,Saheb N, Coulombe MA, Alary ME, Corriveau MP, Authier S et al. Emergence of fluoroquinolones as the predominant risk factor for Clostridium difficile-associated diarrhea: a cohort study during an epidemic in Quebec.Clin Infect Dis. 2005:41(9):1254-60.

Cite this article : Mansoor K, Tanvir SB, Shariq A, Shahnawaz S, Ahmed S. Prevalence and antimicrobial susceptibility pattern of Mono therapy and combination therapy of Cefepime in Pseudomonas aeruginosa isolates of patients from a tertiary care hospital in Karachi, Int. J. Med. Public Health, 2016; 6(3):117-20. 